\title{
MINFLUX: next generation access to the nanoscale
}

\section{Francisco Balzarotti}

\section{Research Institute for Molecular Pathology, Vienna, Wien, Austria}

Superresolution microscopy methods such as STED and PALM/STORM have revolutionized far-field optical fluorescence microscopy by manipulating state transitions of the emitters, offering potentially unlimited resolution. In practice, however, the resolution of an image is limited by the finite photon budget of fluorescent probes, while their finite emission rate imposes a spatial-temporal trade-off in tracking applications. By synergistically combining the strengths of both superresolution families, the recent MINFLUX concept [1] tackles these limitations by rendering each emitted photon more informative.

MINFLUX localizes an emitter by repeatedly probing its location with an excitation beam that features a zero of intensity (fig. 1A-B). The emitter position is obtained from the knowledge of the beam shape and the number of photons collected at each location of the beam. When compared to conventional centroidlocalization techniques (that utilize with the shape of the emitted light), it is possible to reach a given precision by using fewer photons, or conversely, have an improved precision for the same photon budget. Imaging and tracking [1-2] implementations of the concept (fig. 1C-E) demonstrated (i) a 22-fold reduction of the required photon detections, (ii) increased the temporal resolution and the number of localizations per track by 100-fold and (iii) resolved resolving molecules $6 \mathrm{~nm}$ apart with $\sim 1 \mathrm{~nm}$ precision. MINFLUX can also be applied in arbitrarily large regions, allowing imaging in fixed and living cells [3]. This is accomplished by iteratively approaching (fig 2A-D) each photo-activated emitter with a set of MINFLUX localizations, while gradually shrinking the probed region size. This allows isotropic localization precision and surpasses the typical $\propto \mathrm{N}^{-1 / 2}$ dependence, as photons are made increasingly informative as they are acquired. The example (fig. 2E) shows a high power dependence of the precision $\left(\propto \mathrm{N}^{-2}\right)$ with the collected photon number. Additionally, this MINFLUX embodiment operates in three dimensions and for multi-color recordings. Further advances and extensions of the concept will be presented.

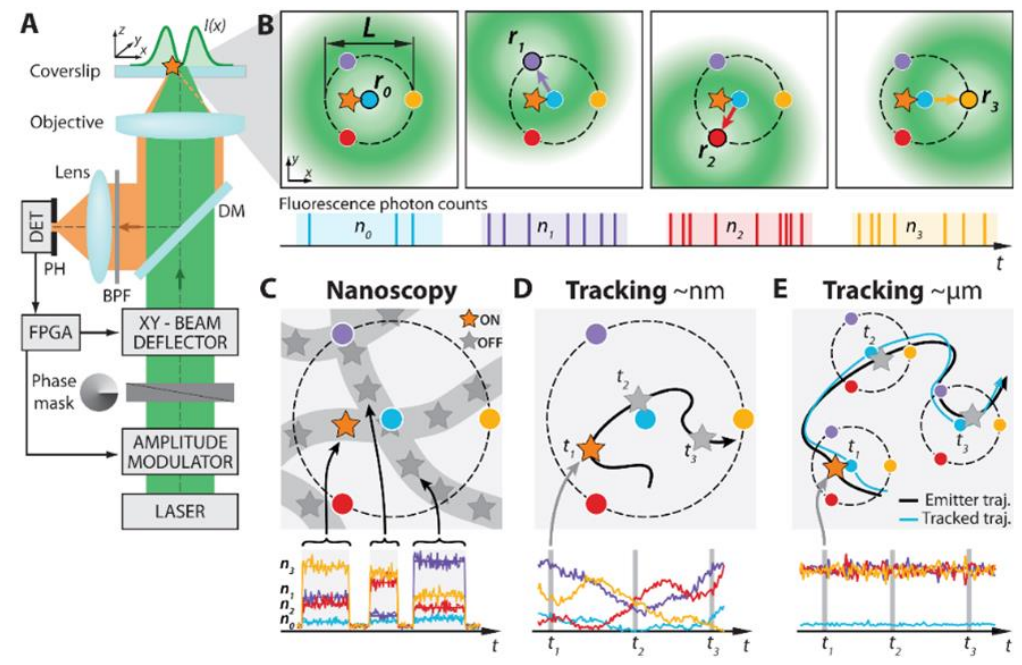

Figure 1. MINFLUX concept. (A) Scanning fluorescence microscope with a donut-shaped excitation beam and confocal detection. (B) An emiter (star) is exposed secuentially to the excitation beam at four different locations, collecting different fluorescence signals. (C-E) Applications of the concept for (C) imaging, by consecutively localizing blinking molecules; (D) nm-range tracking by performing localization very fast; (E) $\mu \mathrm{m}$-range tracking, by reposition of excitation beam pattern onto the emitter with a feedback loop. 


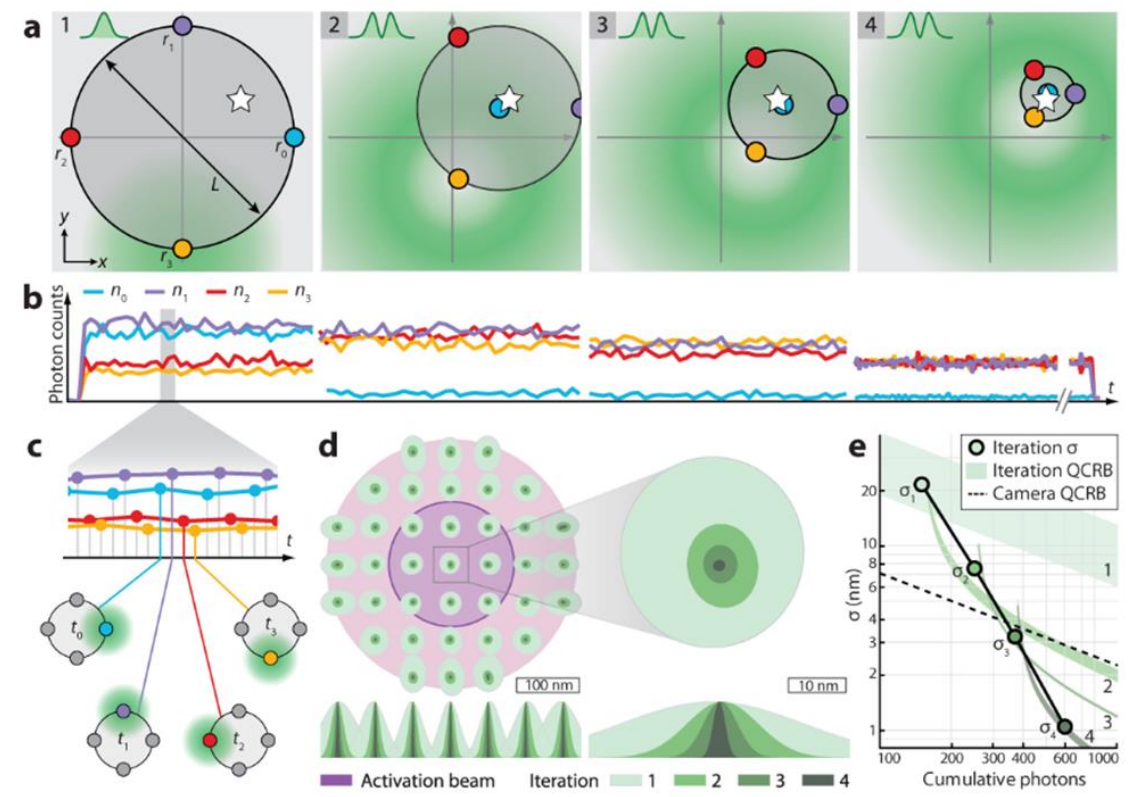

Figure 2. Iterative MINFLUX. (A) Iterative -localization by targeting the beam to four designated coordinates (blue, purple, red, yellow and beam on yellow position in green). Step 1: Gaussian beam, steps 2-4: Doughnut. (B) Typical fluorescence counts per iteration. (C) Representation of the interleaved measurement. (D) Convergence of iterative localizations for molecules within the activation area. (F) Progression of the localization precision - for each iteration (green dots) with the corresponding CRBs (green shades) and the CRB for camera-based localization (dashed line).

\section{References}

[1] F. Balzarotti et al., "Nanometer resolution imaging and tracking of fluorescent molecules with minimal photon fluxes," Science, vol. 355, no. 6325, pp. 606-612, 2017.

[2] Eilers, H. Ta et al., "MINFLUX monitors rapid molecular jumps with superior spatiotemporal resolution.," Proc. Natl. Acad. Sci. U.S.A., p. 201801672, 2018

[3] K. C. Gwosch, J. K. Pape, F. Balzarotti et al., "MINFLUX nanoscopy delivers multicolor nanometer 3D-resolution in (living) cells," bioRxiv, p. 734251, 2019. 